\title{
Analysis of polarization offsets observed for temperature-graded ferroelectric materials
}

\author{
Hui Chen, Taimin Cheng, Hanlei Zheng and Xinxin Zhang \\ Mathematics and physics department, Shenyang University of Chemical Technology, Shenyang
} 110142 ,China

A transverse Ising model in the framework of the mean field approximation is developed to analyze the polarization offsets phenomena in temperature-graded ferroelectric materials. A function of two-spin exchange interaction strength has been introduced to describe the ferroelectric distortion due to the distribution of temperature gradients in materials. Comparisons of the computational results with the experimental data reveal some fundamental factors in the formation of polarization offsets. It is shown that ferroelectric distortion has influenced much on polarization offsets in temperature-graded ferroelectric materials. When quantum fluctuation effect as well as ferroelectric distortion is considered, we have successfully reproduced the experimental observations qualitatively, especially for the indistinguishable polarization offsets from the background at small temperature gradients, which were not successfully reproduced in prior theoretical studies.

PACS: 77.80.Bh; 77.55. $+f ; 77.80 . D j$

The ferroelectric material with polarization gradients normal to the growth surface readily form when gradients in temperature, strain, or composition are coupled 
to the polarization vector in ferroelectric materials. Because of their unconventional ferroelectric properties, they present dramatic hysteresis loop offsets, transpacitor behavior, enormous pyroelectric responses, and other exotic phenomenon [1-20], which have attracted a lot of attention recent these years.

A series of investigations were carried on to interpret the origin of the aberrant hysteretic behavior persist [1-6]. It was experimentally shown that both "up" and "down" hysteresis offsets can be observed from $\left(\mathrm{Ba}_{0.75} \mathrm{Sr}_{0.25}\right) \mathrm{TiO}_{3}$ bulk ceramics [1]. In this ground-breaking experiment a temperature gradient was established along the polarization direction and yielded highly nonlinear polarization offsets for temperatures near the Curie point. Based on the data retrieved from this experiment, a phenomenological approach was subsequently developed to reproduce the experimental observations [2]. The results were in excellent quantitative agreement with the experiment except the data for small temperature gradients. The experimentally determined charge offsets are indistinguishable from the background variation with small temperature difference between two heat sinks [1], while the theoretical results are quite distinguishable [2]. Also with the available experimental data, a microscopic insight was recently put into the polarization response in this temperature-graded alloy through an accurate first-principle-based computational approach [19]. The maximum value of polarization offset was successfully reproduced, but more detailed comparison was not carried on due to the structural and dimensional differences between the samples used in computations and experiment.

These works have (1) discussed the possible "origin" leading to hysteresis 
offsets, (2) explored the fundamental understanding of some exotic properties, (3) demonstrated the potential applications of temperature-graded ferroelectrics as well as some possible ways to tailor them. However, despite all these exciting developments, our understanding of polarization-graded ferroelectrics is still limited. The origin of such polarization offsets is still very much controversial. The indistinguishable charge offsets for small temperature gradients still cannot be reproduced and interpreted theoretically.

The aims of this paper are (1) to gain a fundamental microscopic understanding of polarization offsets for temperature-graded ferroelectrics through a developed transverse Ising model, (2) to theoretically reproduce and interpret the behavior of charge offsets at small temperature gradients in the experiment.

Specifically, due to the structure and dimension of the samples in experiment [1] cannot be accurately reproduced in our model, only qualitative comparisons will be made with the experimental results and other theoretical computations.

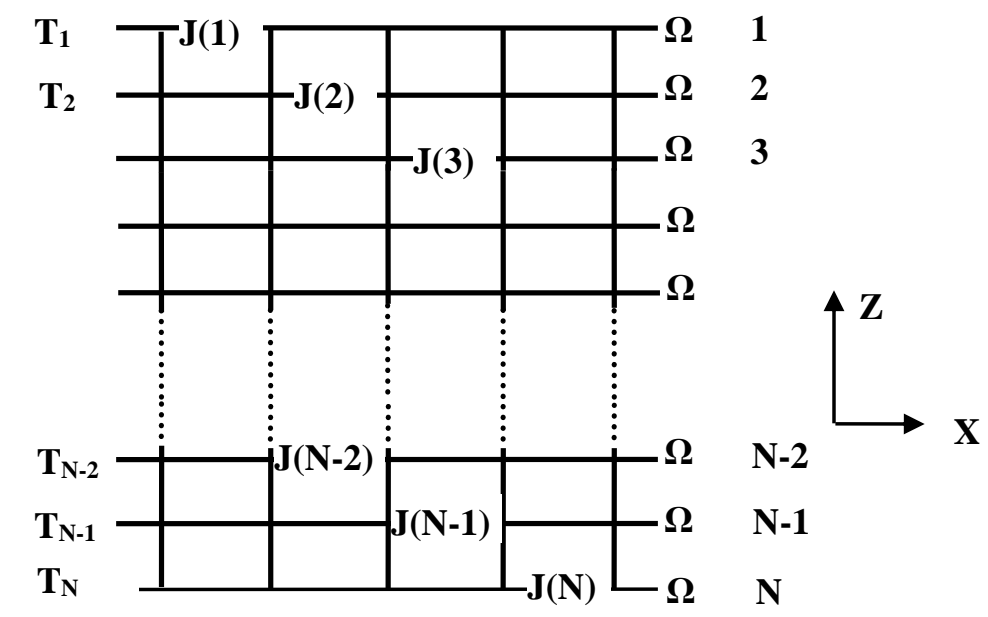

Fig.1 Geometric structure of the temperature-graded ferroelectric material under investigation

The model used to describe the material is illustrated in Fig.1. It is supposed that 
$3 \times 10^{6}$ pseudo-spin layers $\left(N=3 \times 10^{6}\right)$ are contained in the film. The ferroelectric material is imposed a temperature gradient across it, and $T_{1}, T_{2} \ldots T_{N}$ are the temperatures in each pseudo-spin layers. It is assumed that a steady state for heat transfer is established. The easy axis of polarization is along the $z$-axis, and the ferroelectric is considered to be chemically and thermally homogeneous along the $x$ and $y$-directions, reducing the problem to only one dimension. Accordingly, the Hamiltonian of the TIM is

$$
H=-\sum_{i} \Omega_{i} S_{i}^{x} \frac{1}{2} \sum_{i j} J_{i j} \zeta_{i}
$$

where $\Omega_{i}$ is tunneling frequency at site $i, s_{i}^{x}$ and $s_{i}^{z}$ are the $x$ and $z$ components of pseudo-spin at site $i$ (the thermal average of $s_{i}^{z}$ is related to the polarization), $J_{i j}$ is the two-spin exchange interaction strength between site $i$ and site $j$, and the $\operatorname{sum} \sum_{i j}$ runs over all sites. We take $J_{i j}$ to be non-zero only for the nearest-neighbor sites $i$ and $j$.

Quantum fluctuation typically has a very important effect on the structural and thermodynamic properties of materials consisting of light atoms such as hydrogen and helium, and the cubic perovskites, which can exhibit a decisive quantum fluctuation effect despite the fact that the lightest constituent is oxygen. This can occur because these materials have several competing structures with very small structural and energetic differences.

Zhong and Vanderbilt [21] have studied the effects of quantum fluctuation on structural phase transitions in $\mathrm{SrTiO}_{3}$ and $\mathrm{BaTiO}_{3}$. In case of $\mathrm{SrTiO}_{3}$, the quantum fluctuations suppress the ferroelectric phase transition completely. For $\mathrm{BaTiO}_{3}$, the 
quantum effects do not affect the order of the transition, but do reduce the transition temperature by $35-50 \mathrm{~K}$. Furthermore, they also proved that the quantum fluctuation increases with decreasing temperature. In our investigation, the temperatures at small temperature gradients region are not very high, where the fluctuation effects are active and necessary to be considered.

Subsequently, a study of the quantum effect in $\mathrm{BaTiO}_{3}$ was carried on by Zhang etc.[22]. The tunneling frequency was adopted to reflect the impact of quantum fluctuation on the graded films, which was supposed as [22]

$$
\Omega_{m}=g \Omega_{T_{0}}\left(1-\frac{T_{m}}{T_{0}}\right)+\Omega_{T_{0}}
$$

where $\Omega_{m}$ is assumed to be uniform for each layer, $g$ is a parameter indicating the strength of quantum fluctuation and $\Omega_{T 0}$ is the tunneling frequency at the actual Curie-Weiss temperature $T_{0}$ for the ferroelectric material considered. Equation (2) demonstrates that the tunneling frequency depends on the temperature of the specific layer, which varies from layer to layer when the temperature gradients are present.

According to Eq.(2), the tunneling frequency $\Omega_{m}$ of the $m$ th layer will increase linearly from $\Omega_{T_{0}}$ to $(g+1) \Omega_{T_{0}}$ when the temperature decreases from $T_{0}$ to $0 \mathrm{~K}$. Thus, the tunneling frequency $\Omega_{m}$ represents the contribution of the quantum fluctuation.

Zhang et al [22] have given a qualitative analysis for equation (2). For $\mathrm{BaTiO}_{3}$, an eight-site potential exists around the Ti ion. The height $h$ of the potential barrier is finite. When $k_{B} T_{i}$ is higher than the height $h$, the tunneling effect does not exist, which means $\Omega_{m}=0$. For low temperature with $K_{B} T_{i}<h$, the tunneling effect exists, 
implying $\Omega_{m}>0$.

Since the quantum fluctuation effects are considered by Eq.(2) in this paper, its physical implication may restrict our calculations to that kinds of ferroelectric with the one order-disorder phase transition and tunneling vibrational mode (small ions such as protons), but the conclusions we drawn should have the generality.

It is well known that objects expand when heated and contract when cooled. When temperature gradients are established, due to the ununiform temperature distribution, some parts inside the sample may expand heavily, some parts may expand tinily, and some parts even contract where temperatures are very low. Thus, the deformations inside are not synchronous, and the ferroelectric distortions will certainly happen.

However, the ferroelectrics distortions can modify the interaction strength $J$ and the dipolar moment $\mu[22,23]$. As it is quite difficult to accurately model the combined electromechanical setup without introducing a number of adjustable free parameters, we suppose the dipolar moment $\mu$ is constant. A function of strength $J$ was introduced to generally describe the ferroelectric distortions in the sample $[22,23]$.

$$
J(m)=J_{0}\left[1+F_{2} P_{m}^{2}+F_{4} P_{m}^{4}+F_{6} P_{m}^{6}+\cdots\right]
$$

where $m$ is the sequence number of the pseudo-spin layers. $F_{n}(n=2,4,6, \cdots)$ are the coefficients to describe the contribution of the ferroelectric distortion. When ferroelectrics are clamped, $F_{n}=0$. In free ferroelectrics, $F_{n}$ will deviate from zero because of the modification of the polarization, which were taken to be some 
constants in Ref.[22].

In the experiment, the sample was clamped to keep great contact with the heat sinks. According to Ref.[22], $F_{n}$ should taken to be zero. But when considering the ununiform temperature distribution inside the films, the nonsynchronous deformations will certainly happen even in the clamped boundary condition. In this case, the consideration of the statistic distributions with temperatures with respect to these coefficients should be realistic and significant. Hence, we modify the function for strength $J$ to the following form,

$$
\begin{aligned}
& J(m)=J_{0}\left[1+F_{2} e^{-A} P_{m}^{2}+F_{4} e^{-B} P_{m}^{4}+F_{6} e^{-C} P_{m}^{6}\right] \\
& A=\left|J_{0}\left(1+F_{2} P_{m}^{2}\right) / K_{B} T\right| \\
& B=\left|J_{0}\left(1+F_{2} P_{m}^{2}+F_{4} P_{m}^{4}\right) / K_{B} T\right| \\
& C=\left|J_{0}\left(1+F_{2} P_{m}^{2}+F_{4} P_{m}^{4}+F_{6} P_{m}^{6}\right) / K_{B} T\right|
\end{aligned}
$$

The terms higher than the sixth order in the interaction expression are neglected and we take $F_{2}=1.0 \mathrm{~m}^{4} / \mathrm{c}^{2}, F_{4}=10 \mathrm{~m}^{8} / \mathrm{c}^{4}, \quad F_{6}=-10^{3} \mathrm{~m}^{12} / \mathrm{c}^{6}$ as representatives in calculations.

The distribution of temperature gradient in temperature-graded ferroelectrics is very complicated, which is influenced by many factors, such as, the sample thickness, heat-flux density, and thermal-conductivity coefficient etc. [4]. The nonequilibrium molecular dynamics analogous to the experimental method was previously utilized to simulate this thermal transport property, in this case the temperature varies linearly along the sample [19]. Hence a simple uniform temperature gradient is supposed in our calculations, which was adopted in many theoretical works [7,8,9]. Parameter $a$ is introduced to reflect the uniform temperature gradient. Numerically, it is the 
temperature ratio of the $N$ th layer (bottom layer) temperature $T_{N}$ and the first layer (top layer) temperature $T_{1}$, that is,

$$
a=T_{N} / T_{1}
$$

It is worth noting that the temperature gradients are established by changing the temperature of one of the heat sinks, while the latter is kept fixed, which is the case as well as the works in Ref.[1,2,19]. We fix $T_{1}=280 \mathrm{~K}$ in the following calculations.

In order to phenomenologically describe and explain the polarization offsets in temperature-graded ferroelectric materials, it is convenient to rescale the variables into dimensionless forms. We take $\Omega_{T_{0}} / J_{0}=1.2$ and $J_{0} /\left(K_{B} T_{0}\right)=0.1$ as representatives, this particular choice does not affect the generality of the conclusions.
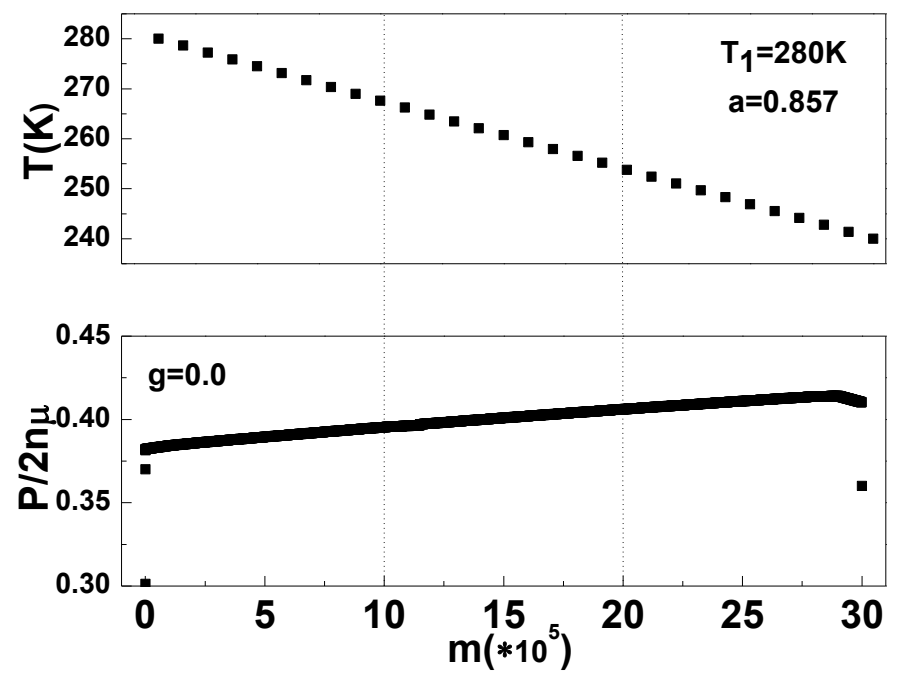

Fig.2 Polarization distribution along the thickness direction of the ferroelectric material

The spontaneous polarizations along the $\mathrm{z}$ direction as a function of sequence number $m$ are plotted in Fig.2. The top panel shows the temperature profile along the $\mathrm{z}$ axis, and the bottom panel shows the polarization distribution associated with this temperature profile. We take $a=0.857$ in the calculation, that is, $\Delta T=40 \mathrm{~K}$. Considering the thickness of the sample $\left(N=3 \times 10^{6}\right)$, the temperature gradient is about 
$10^{4} \mathrm{~K} / \mathrm{m}$, which keeps the same order in the following calculations. The impact of quantum fluctuation is not considered in this figure. It is shown that our results are analogous to the results according to the nonequilibrium molecular dynamics method [19]. The linear temperature difference leads to an almost linear polarization distribution besides at several larger number layers. This result comes out from the competition between the temperature gradient and the interaction couplings strength. Although the last layer has the lowest temperature leading to a larger polarization, the pseudo-spin in the inner layer has a larger coordinate number (six in our case, the coordinate number in the thirtieth layer is five) and a larger indirection. Due to the strong and close interactions between those large number layers and the last one, the weak polarizations extend some depth into inner layers. Finally, this competition leads to the largest polarization in some inner layer.

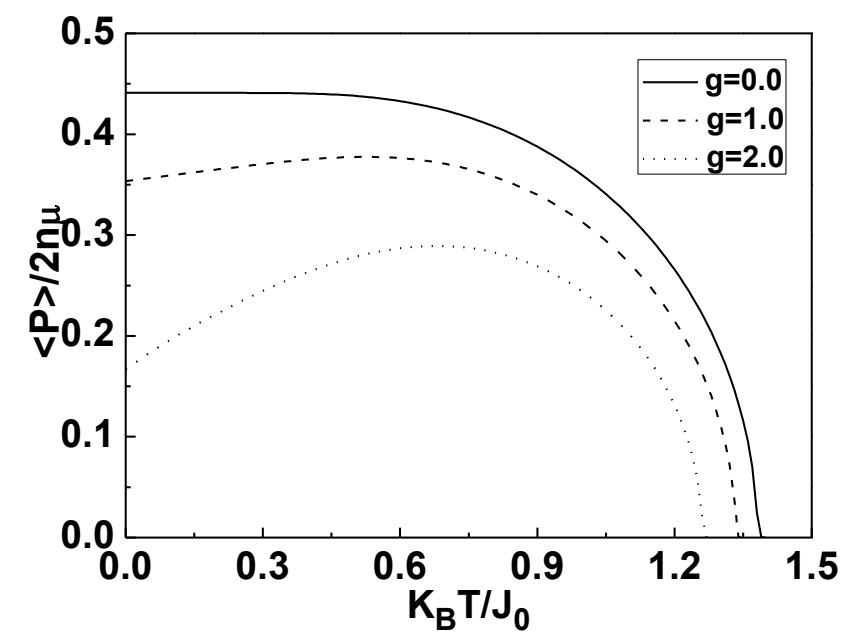

Fig.3 Average spontaneous polarization as a function of temperature for uniform ferroelectric material

The impact of the quantum fluctuation is considered on a uniform ferroelectric material in Fig.3. The average polarization is plotted as a function of temperature. It is 
shown that the quantum fluctuation influences much in polarization, especially when the temperature is not very high. Hence, in following investigation, when temperature-graded materials are concerned, the distribution of quantum fluctuation is inhomogeneous, whose effects will be more obvious in low temperature region. At the same time we can see that the quantum effects do not affect the order of the transition, but do reduce the transition temperatures, which have qualitative agreement with the experimental data in Ref.[1] and the theoretical results in Ref.[9,21].

To eliminate contributions of the boundary conditions and heat sinks, we focus on the region between the $\left(1 \times 10^{6}\right)$ th and the $\left(2 \times 10^{6}\right)$ th layers of the material(the region shown between the two vertical lines in Fig. 2). We will refer to the leftmost and rightmost boundaries of this region as the two sinks, and the difference in temperature between the two sinks is $\Delta T=T_{1 \times 10^{6}}-T_{2 \times 10^{6}}$. Then calculate the polarization offset along the z-axis between the two sinks as Ref.[19] $\Delta P_{z}^{o f f s e t}=P_{z}\left(1 \times 10^{6}\right)-P_{z}\left(2 \times 10^{6}\right)$.

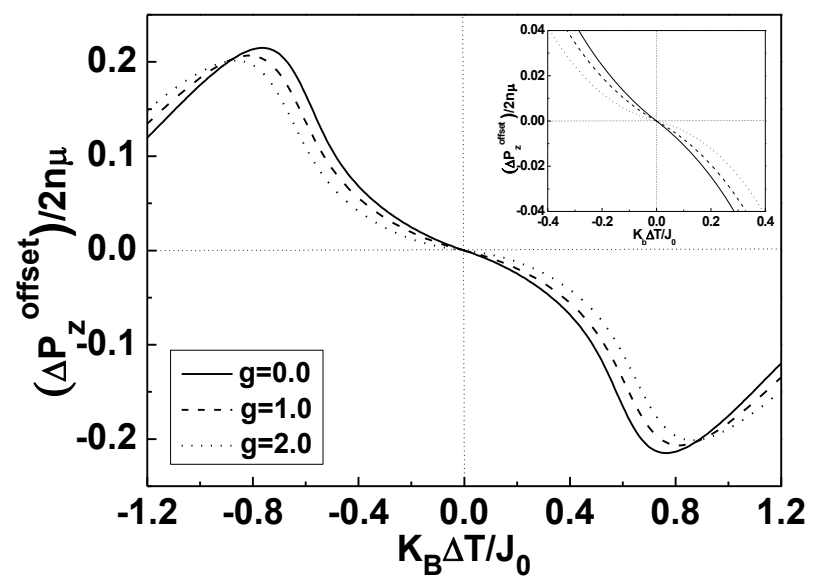

Fig.4 Polarization offset as a function of temperature difference when interaction strength $J$ is constant

In Fig.4 polarization offset is plotted as a function of temperature difference 
when interaction strength $J$ keeps constant. At the same time, the impact of quantum fluctuation is also considered. It is shown that good qualitative agreement is achieved with the former results $[1,2]$. But for small $\Delta T$ regions, where the quantum fluctuation effect is obvious, the offsets still toughly exist, which can be seen from the inserted local magnified figure.

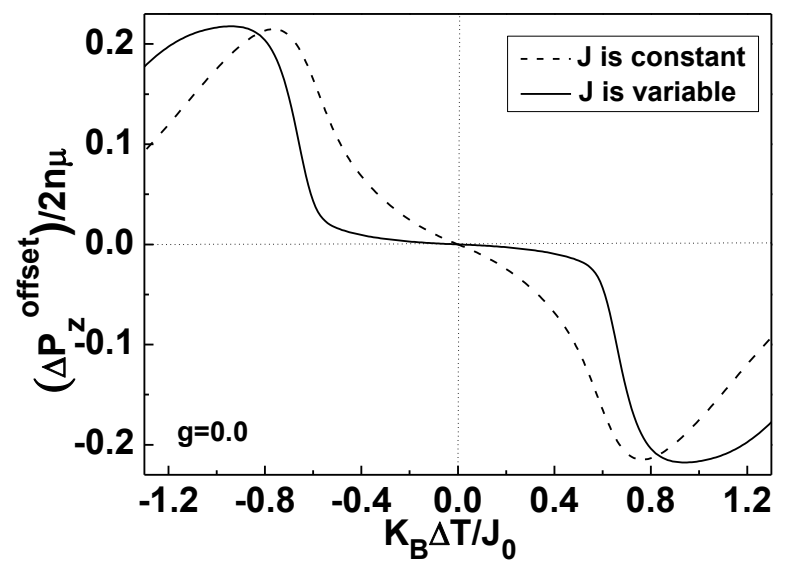

Fig.5 Polarization offset as a function of temperature difference under two conditions:

$\mathrm{J}$ is variable(solid line) and $\mathrm{J}$ is canstant(dashed line)

In Fig.5 the ferroelectric distortion inside due to the temperature gradient is considered( $\mathrm{J}$ is variable), and the result is shown as the solid line. Comparing with the result when the ferroelectric distortion is ignored( $\mathrm{J}$ is constant), which is shown as the dashed line, we can see that the variation of polarization offset with temperature difference is somewhat changed, especially when $\Delta T$ is small. The peak values of charge offsets shift to larger temperature difference region. When $\Delta T$ is small, the dashed charge offsets are very distinct from the background, but the solid charge offsets are not very much distinguishable. When the distortion is ignored, the uniform temperature gradient is the only factor responsible for the charge offsets. However, when the distortion is considered, both the temperature gradient and ferroelectric 
distortion are responsible for the charge offsets, which reflects more realistic situation in the sample. From the nature of the distortion we have pre-articulated, it can be easily accepted that small temperature gradients lead to small distortions, and followed by the small charge offsets. Hence, the ferroelectric distortion can not be ignored, especially when the temperature gradient is not very large, which associated with the obvious change for tiny charge offsets. It is also indicated that the absolute values of charge offsets at small $\Delta T$ regions have greatly decreased, but still exist. From this aspect this problem needs to be further explored.

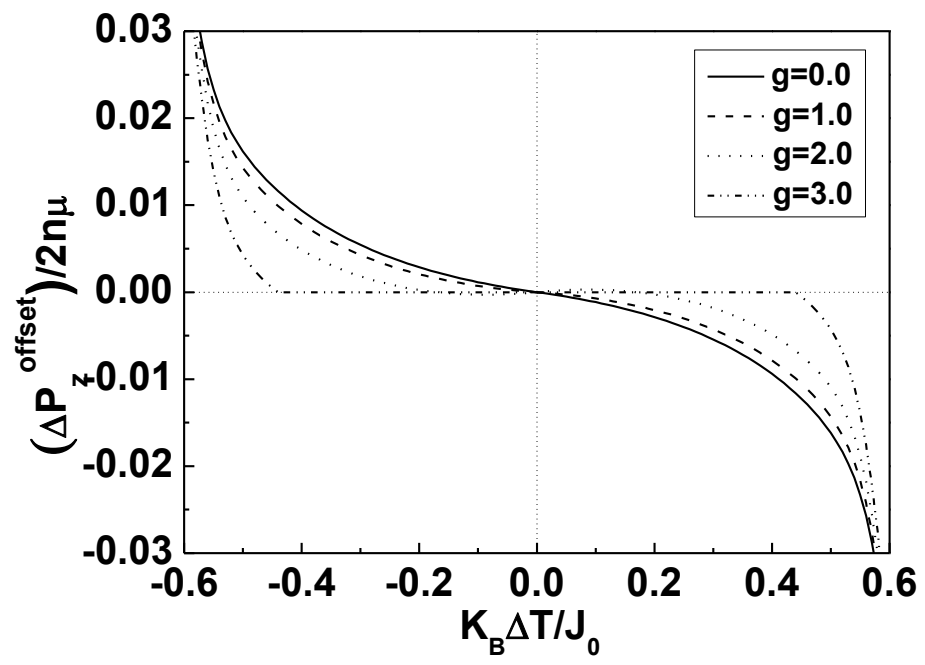

Fig.6 Polarization offset as a function of small temperature difference with different $g$ values when ferroelectric distortion is considered

In Fig.6, the ferroelectric distortions combined with the effect of quantum fluctuation are considered at the same time. It obviously can be seen that when the quantum fluctuation is properly proposed, the charge offsets become indistinguishable from the background, which is in excellent qualitative agreement with the experimental results [1]. Quantum fluctuation effects are active when temperatures are low. With the increasing of the strength of the quantum fluctuation, the charge offsets 
diminish nearly to zero. Hence we can draw the conclusion that both ferroelectric distortion and quantum fluctuation effect are the two important factors to influence the formation of polarization offsets.

It should be noted that our results do not, however, completely qualitatively replicate the shape of the observed polarization offset curve in experiment, which may be explained by the fact that the model we adopted does not capture the full complexity of the ferroelectric material.

\section{Conclusions}

Polarization offset phenomena in temperature-graded ferroelectric material is investigated by means of a transverse Ising model. A function of two-spin exchange interaction strength has been introduced to describe the ferroelectric distortion due to the distribution of temperature gradients in materials. The results have shown that ferroelectric distortion and the quantum fluctuation effect are the two important factors influencing the formation of polarization offsets. Our results qualitatively agreed with the experimental observations. Especially, we have successfully replicated the indistinguishable charge offsets at low temperature gradients, which were not successfully reproduced in former theoretical studies.

\section{Reference}

[1] FellbergW, Mantese J, Schubring N and Micheli A 2001 Origin of the 'up', 'down' hysteresis offsets observed from polarization-graded ferroelectric materials Appl. Phys. Lett. 78 524-6

[2] Zhong S, Ban Z-G, Alpay S P and Mantese J V 2006 Large piezoelectric strains from polarization graded ferroelectrics Appl. Phys. Lett. 89142913

[3] Pintilie L, Boerasu I and Gomes M J M 2003 Simple model of polarization offset of graded ferroelectric structures J. Appl. Phys. 939961

[4] Bogomolov A A, Solnyshkin A V 2005 Crystallograhpy Report 50 S53

[5] Chan H K, Lam C H and Shin F G 2004 Time-dependent space-charge-limited conduction as a 
possible origin of the polarization offsets observed in compositionally graded ferroelectric films J. Appl. Phys. 952665

[6] S. Zhong, S. P. Alpay, andZ.-G. Ban, 2005 Effective pyroelectri response of compositionally graded ferroelectric materials Appl. Phys. Lett. 86092903

[7] Alpay S P, Ban Z-G and Mantese J V 2003 Thermodynamic analysis of temperature-graded ferroelectrics Appl. Phys. Lett. 821269

[8] Cao H X, Lo V C and Li Z-Y 2005 Dielectric response of temperature-graded ferroelectric films $J$. Appl.Phys. 98114105

[9] Cao H X and Zhen Y L 2003 Thermodynamic properties of temperature-graded ferroelectric film J.Phys.: Condens.Matter 156301

[10] Cao H X, Gao Y-H, Jiang Q and Li Z-Y 2004 Thermodynamic properties of compositionally graded $\mathrm{Ba}_{-x}-\mathrm{Sr}_{x} \mathrm{TiO}_{3}$ thin films J. Appl. Phys. 961628

[11] Zhang J, Tang M H and Tang J X et al. 2007 Bilayer model of polarization offset of compositionally graded ferroelectric thin films Appl. Phys. Lett. 91162908

[12] Zhong S, Alpay S P, Ban Z-G and Mantese J V 2005 Effective pyroelectric response of compositionally graded ferroelectric materials Appl. Phys. Lett. 86092903

[13] Mantese J V, Schubring NWand Micheli A L 2002 Polarization-graded ferroelectrics: transpacitor push-pull amplifier Appl. Phys. Lett. 801430

[14] Marvan M, Chvosta P and Fousek J 2005 Theory of compositionally graded ferroelectrics and pyroelectricity Appl. Phys. Lett. 86221922

[15] Zhou Y, Chan H K, Lam C H and Shin F G 2005 Effects of polarization and permittivity gradients and other parameters on the anomalous vertical shift behavior of graded ferroelectric thin films $J$. Appl. Phys. 98034105

[16] Artemev A, Geddes B, Slutsker J and Roytburd A 2008 Thermodynamic analysis and phase field modeling of domain structures in bilayer ferroelectric thin films J. Appl. Phys. 103074104

[17] Okatan M B, Mantese J V and Alpay S P 2009 Polarization coupling in ferroelectric multilayers Phys. Rev. B 79174113

[18] Zhou Y 2012 Enhancement of photovoltaic effect in nanoscale polarization graded ferroelectrics Solar Energy 86 811-5

[19] Zhang Q and Ponomareva I 2010 Microscopic insight into temperature-graded ferroelectrics Phys. Rev. Lett. 105147602

[20] Zhang Q and Ponomareva I 2013 Depolarizing field in temperature-graded ferroelectrics from an atomistic viewpoint New J. Phys. 15043022

[21] Zhong W L and Vanderbilt D 1996 Effect of quantum fluctuations on structural phase transitions in $\mathrm{SrTiO}_{3}$ and $\mathrm{BaTiO}_{3}$ Phys. Rev. B 535047

[22] Zhang L, Zhong W and Kleemann W 2000 A study of the quantum effect in $\mathrm{BaTiO}_{3}$ Phys.Lett.A 276162

[23] Zhang L and Zhong W L 1999 Order-disorder model in ferroelectrics Phys.Lett.A 260279

Acknowledgements

This work was supported by the National Science Foundation of China under grant No.11374215 and the Educational Scientific Research Project of Liaoning Province under grant No. L2014172. 